PROCEEDINGS OF THE

AMERICAN MATHEMATICAL SOCIETY

Volume 129, Number 6, Pages 1581-1585

S 0002-9939(00)05742-7

Article electronically published on October 31, 2000

\title{
DEPTH OF SYMMETRIC ALGEBRAS OF CERTAIN IDEALS
}

\author{
MARK R. JOHNSON
}

(Communicated by Wolmer V. Vasconcelos)

\begin{abstract}
We compute the depth of the symmetric algebra of certain ideals in terms of the depth of the ring modulo the ideal generated by the entries of a minimal presentation matrix.
\end{abstract}

The purpose of this paper is to give a formula for the depth of the symmetric algebra $S(I)=\bigoplus_{j \geq 0} S_{j}(I)$ for certain ideals. We also obtain an analogous result for the symmetric algebra $S\left(I / I^{2}\right)$ of the conormal module (as an $R / I$-module). Our method is a simple application of the recent results $([3],[5])$ concerning the Cohen-Macaulay properties of the blow-up rings $R[I t]=\bigoplus_{j \geq 0} I^{j}$ and $\operatorname{gr}_{I}(R)=$ $\bigoplus_{j \geq 0} I^{j} / I^{j+1}$. In particular, these blow-up rings are Cohen-Macaulay for the ideals under our consideration. It is well known that the symmetric algebra need not be Cohen-Macaulay, but it turns out that these symmetric algebras have fairly large depth in any case.

Let $R$ be a noetherian local ring with infinite residue field $k$, and let $I$ be an ideal. Recall that the analytic spread of $I$ is $\ell(I)=\operatorname{dim} R[I t] \otimes_{R} k$, or equivalently is the least number $s$ of elements $a_{1}, \ldots, a_{s}$ in $I$ such that $I^{k+1}=\left(a_{1}, \ldots, a_{s}\right) I^{k}$ for some $k \geq 0$; the least integer $k$ (over all such reductions) is the reduction number $r$ of $I$. Note that $r=0 \Longleftrightarrow \ell=n \equiv \nu(I)$ (the minimal number of generators of $I$ ) $\Longleftrightarrow I$ is generated by analytically independent elements. We say that $I$ satisfies $G_{s}$ if $\nu\left(I_{p}\right) \leq \operatorname{dim} R_{p}$ whenever $p \in V(I)$ and $\operatorname{dim} R_{p}<s$, and that $I$ satisfies $G_{\infty}$ if $I$ satisfies $G_{s}$ for every $s$. We will also need the Artin-Nagata property [7]: $I$ satisfies $A N_{s}$ (respectively $A N_{s}^{-}$) if $J=\left(a_{1}, \ldots, a_{s}\right): I$ is Cohen-Macaulay whenever $a_{1}, \ldots, a_{s} \in I$ and $h t J \geq s \geq h t I$ (respectively, and $h t I+J>s$ ).

Theorem 1. Let $R$ be a local Cohen-Macaulay ring with infinite residue field, let $I$ be an ideal with $h t I \geq 2$, analytic spread $\ell$, reduction number $r$, minimally generated by $n=\ell+1$ elements, let $\phi$ be a minimal presentation matrix of $I$, and assume that $I$ satisfies $G_{\ell}$ and $A N_{\ell-2}^{-}$, and that $S_{j}(I) \cong I^{j}$ and $\operatorname{depth} R / I^{j} \geq$ $\operatorname{dim} R-\ell+r-j$ for $1 \leq j \leq r$. Then

$$
\begin{gathered}
\operatorname{depth} S(I)=\operatorname{depth} R / I_{1}(\phi)+n, \\
\operatorname{depth} S\left(I / I^{2}\right)=\min \left\{\operatorname{depth} R / I_{1}(\phi)+n, \operatorname{dim} R\right\} .
\end{gathered}
$$

Received by the editors June 17, 1999 and, in revised form, September 1, 1999.

1991 Mathematics Subject Classification. Primary 13A30, 13H10.

(C)2000 American Mathematical Society 
Proof. By the universal property of the symmetric algebra, there exists a canonical exact sequence

$$
0 \longrightarrow \mathcal{A} \longrightarrow S(I) \longrightarrow R[I t] \longrightarrow 0 .
$$

However, by [3, Theorem 2.6 and Lemma 3.1], $\mathcal{A}$ is cyclic, and is annihilated by $I_{1}(\phi)$. It then follows easily that $\operatorname{Ann}(\mathcal{A})=I_{1}(\phi) S(I)$. Hence $\mathcal{A} \cong S(I) / \operatorname{Ann}(\mathcal{A}) \cong$ $S(I) / I_{1}(\phi) S(I) \cong R / I_{1}(\phi) \otimes_{R} S(I)$. Now let $S=R\left[T_{1}, \ldots, T_{n}\right]$. Then there is an epimorphism $S \longrightarrow S(I)$ whose kernel $L$ is generated by all linear relations on $I$. Hence then there is an $S$-module isomorphism $\mathcal{A} \cong R / I_{1}(\phi) \otimes_{R} S / L \cong R / I_{1}(\phi) \otimes_{R} S$, and thus we obtain an exact sequence

$$
0 \longrightarrow R / I_{1}(\phi) \otimes_{R} S \longrightarrow S(I) \longrightarrow R[I t] \longrightarrow 0 .
$$

Tensoring with $R / I$, we obtain the corresponding exact sequence

$$
0 \longrightarrow R / I_{1}(\phi) \otimes_{R} S \longrightarrow S\left(I / I^{2}\right) \longrightarrow g r_{I}(R) \longrightarrow 0 .
$$

Now [5. Theorem 3.1 and Corollary 3.4] shows that the rings $R[I t]$ and $g r_{I}(R)$ are Cohen-Macaulay. Note that $\operatorname{dim} S(I)=\operatorname{dim} R+1$, while $\operatorname{dim} S\left(I / I^{2}\right)=\operatorname{dim} R$ if and only if $I$ satisfies $G_{\infty}$, and otherwise $\operatorname{dim} S\left(I / I^{2}\right)=\operatorname{dim} R+1$ (11, Proposition $6.5]$ ). Thus the first formula is immediate, and the second one will follow once we show that depth $S\left(I / I^{2}\right) \leq \operatorname{dim} R$. Hence supposing that depth $S\left(I / I^{2}\right)>$ $\operatorname{dim} R$, necessarily $S\left(I / I^{2}\right)$ must be Cohen-Macaulay of dimension $\operatorname{dim} R+1$, and $I$ does not satisfy $G_{\infty}$. In particular, $S\left(I / I^{2}\right)$ is (locally) equidimensional. However, one of its minimal components will be that of the associated graded ring, and $\operatorname{dim} \operatorname{gr}_{I}(R)=\operatorname{dim} R$. This contradiction completes the proof.

As a corollary we find that, with our current assumptions, the two symmetric algebras can never be simultaneously Cohen-Macaulay.

Corollary 1. With the assumptions of Theorem 1 ,

$S(I)$ is Cohen-Macaulay if and only if $I_{1}(\phi)$ is Cohen-Macaulay of height $n-1$, $S\left(I / I^{2}\right)$ is Cohen-Macaulay if and only if $I_{1}(\phi)$ is Cohen-Macaulay of height $n$.

Proof. If $I_{1}(\phi)$ is Cohen-Macaulay of height $n-1$ (respectively $n$ ), then clearly $S(I)$ (respectively $S\left(I / I^{2}\right)$ ) is Cohen-Macaulay. To show the converse, note our assumptions imply that $h t I_{1}(\phi) \geq n-1$ [5. Remark 2.7]. Hence if $S(I)$ is CohenMacaulay, then

$$
\begin{aligned}
\operatorname{dim} R+1 & =\operatorname{dim} S(I)=\operatorname{depth} S(I)=\operatorname{depth} R / I_{1}(\phi)+n \leq \operatorname{dim} R / I_{1}(\phi)+n \\
& =\operatorname{dim} R-h t I_{1}(\phi)+n \leq \operatorname{dim} R+1 .
\end{aligned}
$$

Hence we must have that depth $R / I_{1}(\phi)=\operatorname{dim} R / I_{1}(\phi)$ and that $h t I_{1}(\phi)=n-1$. For $S\left(I / I^{2}\right)$, note that as in the proof of Theorem 1 , if $S\left(I / I^{2}\right)$ is Cohen-Macaulay, then $I$ satisfies $G_{\infty}$; hence $h t I_{1}(\phi) \geq n$. Thus in this case

$$
\begin{aligned}
\operatorname{dim} R & =\operatorname{dim} S\left(I / I^{2}\right)=\operatorname{depth} S\left(I / I^{2}\right)=\min \left\{\operatorname{depth} R / I_{1}(\phi)+n, \operatorname{dim} R\right\} \\
& \leq \min \left\{\operatorname{dim} R / I_{1}(\phi)+n, \operatorname{dim} R\right\}=\min \left\{\operatorname{dim} R-h t I_{1}(\phi)+n, \operatorname{dim} R\right\} \\
& \leq \operatorname{dim} R,
\end{aligned}
$$

and hence we conclude that $R / I_{1}(\phi)$ is Cohen-Macaulay and that $h t I_{1}(\phi)=n$.

Corollary 2. With the assumptions of Theorem 1, assume further that I satisfies $A N_{\ell}$. Then $S(I)$ is Cohen-Macaulay. 
Proof. By [3, Proposition 3.2], after elementary row operations, the entries of one row of $\phi$ generate $I_{1}(\phi)$. Thus $I_{1}(\phi)=\left(a_{1}, \ldots, a_{\ell}\right): a_{\ell+1}=\left(a_{1}, \ldots, a_{\ell}\right): I$, for some generating set $a_{1}, \ldots, a_{\ell+1}$ of $I$. Since $I$ satisfies $A N_{\ell}$, it follows that $I_{1}(\phi)$ is a Cohen-Macaulay ideal and has height exactly $\ell$ [7, Proposition 1.7].

One may view this latter corollary as a partial generalization of one of the main results of [1] (Theorem 6.10), which guarantees that the symmetric algebra is Cohen-Macaulay for ideals satisfying sliding depth.

In our situation we have a good description of the components of the symmetric algebra, as hinted at in the proof of the Theorem. Indeed, under those hypotheses, we have the decomposition $(0)=I_{1}(\phi) S(I) \cap \mathcal{A}$. This is clear since $\mathcal{A}$ is cyclic, generated by a form not contained in the extension of the maximal ideal, and has annihilator $I_{1}(\phi) S(I)$.

Corollary 3. With the assumptions of Theorem 1,

$S(I)$ is unmixed if and only if $I_{1}(\phi)$ is unmixed of height $n-1$,

$S\left(I / I^{2}\right)$ is unmixed if and only if $I_{1}(\phi)$ is unmixed of height $n$.

Corollary 4. With the assumptions of Theorem 1, assume further that I satisfies $A N_{\ell-1}^{-}$. Then $S(I)$ is unmixed.

Proof. As in the proof of Corollary 2, $I_{1}(\phi)$ is unmixed of height $\ell$ by [7, Proposition 1.7].

We now are able to answer a question of Herzog, Simis and Vasconcelos [2, p.142]. They asked whether there is a Cohen-Macaulay (generic complete intersection) ideal for which $S\left(I / I^{2}\right)$ is Cohen-Macaulay and $S(I)$ is not. (Non-Cohen-Macaulay ideals with this property were known.) It is known [1, Corollary 6.9] that such an ideal must necessarily satisfy $G_{\infty}$. Recently, an example was found in [6, Example 6.15] but the ring $R$ is not regular, which, as they mention, still leaves the question open in this case.

Corollary 1 now gives an answer to this question in principle. To show that such examples exist in great number, we will state the result in a less technical manner.

Corollary 5. Let $R$ be a local Gorenstein ring of dimension $d$, and let $I$ be a Cohen-Macaulay ideal of grade $g \geq 2$ satisfying $G_{\infty}$ and $\operatorname{depth} R / I^{j} \geq d-g-j+1$ for $1 \leq j \leq d-g-2$ and suppose that $\ell(I)<d$. Then $S\left(I / I^{2}\right)$ is Cohen-Macaulay, but $S(I)$ is Cohen-Macaulay if and only if $\nu(I)=\ell(I)$.

Proof. We may assume that the residue field is infinite. If $n=\nu(I)<d$, then by 7, Corollary 2.13] $I$ is strongly Cohen-Macaulay; hence $S\left(I / I^{2}\right)$ (as well as $S(I)$ ) is Cohen-Macaulay by [2, Theorem 2.6]. This Theorem also shows that $S(I) \cong R[I t]$; hence in particular $n=\ell$. Thus we may assume that $n=d$.

We now claim that $\ell=n-1$. For otherwise, suppose that $\ell \leq n-2$. Our assumption on the depth of the powers of $I$ implies that $I$ satisfies $A N_{d-3}$ [7] Theorem 2.9], and hence in particular $A N_{\ell-1}$. Let $\left(a_{1}, \ldots, a_{\ell}\right) \subset I$ be any reduction of $I$. Then $h t J=\left(a_{1}, \ldots, a_{\ell}\right): I \geq \ell+1$ by [7, Proposition 1.11]. However, as $I$ satisfies $A N_{\ell-1}, J$ must have height exactly $\ell$ by [7, Proposition 1.7]. This contradiction proves the claim.

Thus we now have that $n=d=\ell+1$ and that $I$ satisfies $A N_{\ell-2}$. But now [7] Theorem 4.1] (or [5, Proposition 4.7]) gives that $r(I)=1$. Thus $I$ satisfies all the hypotheses of Theorem 1 . Since $h t I_{1}(\phi)=d$, the result follows immediately from Corollary 1. 
We conclude with several examples.

Example 1. Let $(R, m)$ be a 6 -dimensional regular local ring, and let $X$ be a $2 \times 4$ matrix such that $I_{1}(X)=m$ and $h t I_{2}(X) \geq 3$. Then $I=I_{2}(X)$ is a perfect ideal of grade 3 , a complete intersection on the punctured spectrum, $S\left(I / I^{2}\right)$ is Cohen-Macaulay, and $S(I)$ is not Cohen-Macaulay.

Proof. It is well known that $I$ is a perfect ideal of grade 3 , and it is readily seen to be a complete intersection on the punctured spectrum. As $n=\nu(I)=6=\operatorname{dim} R$, in particular $I$ satisfies $G_{\infty}$. Since the well-known Plücker relation on the minors of $X$ shows that $\ell<n$, the result follows from Corollary 5 .

One may even achieve that $I$ is an isolated singularity. For example, if $x_{1}, \ldots, x_{6}$ is a regular system of parameters, one may take $I=I_{2}\left(\begin{array}{llll}x_{1} & x_{2} & x_{3} & x_{4} \\ x_{5} & x_{6} & x_{1} & x_{2}\end{array}\right)$.

The next example is obtained by linking a certain tensor product, as in [4].

Example 2. Let $A=k\left[\left[x_{1}, \ldots, x_{d}\right]\right]$, and let $J$ be an ideal such that $A / J$ is CohenMacaulay, of dimension 2 and type 2, and a complete intersection in codimension one. Let $\underline{\alpha}=\alpha_{1}, \ldots, \alpha_{g}$ be a regular sequence in $J$ which generates $J$ generically. Consider the isomorphism $\phi: k\left[\left[x_{1}, \ldots, x_{d}\right]\right] \longrightarrow k\left[\left[y_{1}, \ldots, y_{d}\right]\right]$ given by $\phi\left(x_{i}\right)=y_{i}$, let $J^{\prime}$ and $\underline{\alpha}^{\prime}$ be the image of $J$ and $\underline{\alpha}$ under $\phi$, and set $R=k\left[\left[x_{1}, \ldots, x_{d}, y_{1}, \ldots, y_{d}\right]\right]$ and $I=\left(\underline{\alpha}, \underline{\alpha^{\prime}}\right) R:\left(J, J^{\prime}\right) R$.

Then $I$ is a perfect ideal of $R$, generically a complete intersection, $S\left(I / I^{2}\right)$ is Cohen-Macaulay, and $S(I)$ is not Cohen-Macaulay.

Proof. The result follows from Corollary 5, using [4, Remark 2.3 and Lemma 2.4].

The simplest example of this occurs when $d=4$ and one takes $J=\left(x_{1} x_{2}, x_{2} x_{3}\right.$, $x_{3} x_{4}$ ). The resulting ideal $I$ is perfect of grade 4 and deviation 4 ; it may be viewed as the edge ideal of a certain graph [4, Example 3.8].

Example 3. Let $(S, m)$ be a 5-dimensional regular local ring containing a field, $X$ an alternating $5 \times 5$ matrix with $I_{1}(X)=m$ and $h t I_{4}(X) \geq 3, Y$ a generic $5 \times 1$ matrix, $R=S[[Y]]$, and $I=P f_{4}(X)+I_{1}(X Y)$.

Then $I$ is a perfect Gorenstein ideal of grade 5, a complete intersection on the punctured spectrum, $S\left(I / I^{2}\right)$ is Cohen-Macaulay, and $S(I)$ is not Cohen-Macaulay.

Proof. In this case we apply Corollary 1, after specializing the generic case, using 7, Theorem 2.14, and Example 2.12].

\section{REFERENCES}

[1] J. Herzog, A. Simis and W. V. Vasconcelos, Approximation complexes of blowing-up rings II, J. Algebra 82(1983), 53-83. MR 85b:13015

[2] J. Herzog, A. Simis and W. V. Vasconcelos, Koszul homology and blowing-up rings, Commutative Algebra (Trento, 1981), 79-169, Lecture Notes in Pure and Appl. Math., 84, Dekker, New York, 1983. MR 84k:13015

[3] M. Johnson, Second analytic deviation one ideals and their Rees algebras, J. Pure Appl. Algebra 119(1997), 171-183. MR 98e:13005

[4] M. Johnson, Linkage and sums of ideals, Trans. Amer. Math. Soc. 350(1998), 1913-1930. MR 98h:13015

[5] M. Johnson and B. Ulrich, Artin-Nagata properties and Cohen-Macaulay associated graded rings, Compositio Math. 103(1996), 7-29. MR 97f:13006 
[6] A. Simis, B. Ulrich and W. V. Vasconcelos, Tangent Star Cones, J. Reine Angew. Math. 483 (1997), 23-59. MR 97m:14001

[7] B. Ulrich, Artin-Nagata properties and reductions of ideals, Commutative algebra: syzygies, multiplicities, and birational algebra (South Hadley, MA, 1992), 373-400, Contemp. Math., 159, Amer. Math. Soc., Providence, RI, 1994. MR 95a:13017

Department of Mathematical Sciences, University of Arkansas, Fayetteville, ARKANSAS 72701

E-mail address: mark@math.uark.edu 